\title{
Ascent of the kappa-opioid receptor in psychopharmacology
}

\author{
William A. Carlezon Jr. • Klaus A. Miczek
}

Received: 24 March 2010/Accepted: 24 March 2010/Published online: 17 April 2010

(C) Springer-Verlag 2010

Often overshadowed by other members of the endogenous opioid receptor family, kappa-opioid receptors (KORs) are rapidly emerging as an important target for the study and treatment of a variety of central nervous system disorders. This Special Issue is dedicated to 18 papers that describe some of the newest and most important developments.

Since the seminal work of Pasternak (1980), there has been great interest in selective KOR agonists for the management of pain. Stimulation of KORs produces analgesia, while the lack of effects at mu-opioid receptors (MORs) minimizes abuse liability. Pain remains a likely indication for KOR agonists (this issue). However, early studies demonstrated that administration of selective KOR agonists to humans elicited unwanted effects including dysphoria, derealization, and depersonalization, some of which can be modeled in animals (this issue). The prominence of these effects has tempered enthusiasm for further development of these agents for clinical use. It is now better understood that MORs and KORs are often located in anatomical apposition within brain areas such as the nucleus accumbens (NAc). As a result of this arrangement, selective activation of these receptor subtypes tends to produce opposite effects on behavior: whereas stimulation of MORs is rewarding, stimulation of KORs causes dysphoria. The work by Negus et al. (this issue)

\footnotetext{
W. A. Carlezon Jr. ( $\square)$

Behavioral Genetics Laboratory, Department of Psychiatry, Harvard Medical School, McLean Hospital, MRC 217, 115 Mill Street,

shows that KOR agonists concurrently reduce pain while producing depressive-like behavior, and provides a novel methodology that could be used to screen for novel analgesics with less propensity to affect mood. Chartoff et al. (this issue) use an interesting new ligand, salvinorin A, to provide a potential mechanism by which KOR agonists cause depressive-like behavior: reduction of phasic dopamine release in the NAc. Salvinorin A is of special interest because it is a potent and highly selective KOR agonist that binds to few (if any) other receptors in the brain. Butelman et al. (this issue) and Nemeth et al. (this issue) show how the unique pharmacological profile of salvinorin A can enable new insights on the brain substrates of the types of altered perception that often accompany psychiatric conditions such as schizophrenia. Bruchas and Chavkin (this issue) provide evidence that in addition to their acute dysphoric effects, KOR agonists mimic the cellular effects of stress. The work of Sperling et al. (this issue) implicates the stress-like effects of KORs in increased vulnerability to drugs of abuse including ethanol. Wee and Koob (this issue) describe how KOR stimulation may play a key role in the addiction cycle by influencing processes of positive and negative reinforcement, tying together the ways in which stress, depression, and addiction can interact to control behavior.

Until recently, the potential for clinical applications of KOR antagonists seemed limited. These agents were originally envisioned as tools for studies of interactions between agonists and KORs and the functional significance of KORs for behavior, or for understanding receptorreceptor interactions (e.g., dimers). Several papers in this issue suggest that KOR antagonists have potential for broad clinical applicability. As one example, Carr and Lucki (this issue) demonstrate that the KOR antagonist DIPPA has acute anxiolytic-like effects in addition to acute antidepressant- 
like effects, a profile that distinguishes this class of drugs from standard antidepressants. Jackson et al. (this issue) show that the KOR antagonists JDTic and norBNI block the physical and motivational signs of nicotine withdrawal in rats; a drug with these effects in humans would have a tremendous impact on health issues ranging from addiction to cancer. Curiously, all three of the KOR antagonists described above (DIPPA, JDTic, and norBNI) share the property of having long-lasting or irreversible actions. This complicates their use in humans, at least at the early stages of drug development, when a short duration of action or the ability to reverse unanticipated side effects would be preferable until the safety of these drugs is established. The fact that non-selective opioid antagonists such as naloxone do not have these long-lasting effects on KOR function raises two questions that are of critical importance for future work: (1) is it even possible to develop a KOR antagonist that is both selective and shortacting, and (2) are the long-lasting effects of the prototypical
KOR antagonists critical for their efficacy in animal models?

Despite these and other exciting advances described in this issue, it is obvious that there is still much to be learned about the role of KORs in processes that interest the readership of Psychopharmacology. If the trajectory of the past decade continues, the next Special Issue on KORs will be considerably thicker than this one.

Disclosure Dr. Carlezon is an inventor on US patents (Assignee: McLean Hospital) related to the use of kappa-opioid receptor ligands in the treatment of psychiatric disorders.

\section{Reference}

Pasternak GW (1980) Multiple opiate receptors: [3H]ethylketocyclazocine receptor binding and ketocyclazocine analgesia. Proc Natl Acad Sci U S A 77:3691-3694 